\title{
On the relationships between some games associated with SUOWA and Semi-SUOWA operators
}

\author{
Bonifacio Llamazares* \\ Departamento de Economía Aplicada, Instituto de Matemáticas (IMUVA), and BORDA Research Unit, \\ Universidad de Valladolid, Avda. Valle de Esgueva 6, 47011 Valladolid, Spain.
}

\begin{abstract}
The construction of functions that simultaneously generalize weighted means and OWA operators is an interesting topic that has received special attention in recent years. Due to the properties they satisfy, one of the most interesting generalization are SUOWA operators, which have been widely studied in the literature. In a recent paper, a new generalization has been introduced, the Semi-SUOWA operators, which have a close relationship with SUOWA operators. The main aim of this paper is to analyze the games associated with Semi-SUOWA operators. In this respect, we give conditions under which we can guarantee the monotonicity of these games. Moreover, we establish some relationships between some games associated with SUOWA and Semi-SUOWA operators and show the pointwise convergence of certain games.
\end{abstract}

Keywords: Choquet integral, SUOWA operators, Semi-SUOWA operators, weighted means, OWA operators.

\section{Introduction}

The search for functions that simultaneously generalize weighted means and ordered weighted averaging (OWA) operators (Yager [1]) is an interesting problem in the field of aggregation operators. Given that both families of functions are defined by means of weighting vectors, most of the studies consider functions parametrized by two weighting vectors, $\boldsymbol{p}$ for the weighted mean and $\boldsymbol{w}$ for the OWA operator, so that we can recover the weighted mean when $\boldsymbol{w}=(1 / n, \ldots, 1 / n)$ and the OWA operator when $\boldsymbol{p}=(1 / n, \ldots, 1 / n)$.

Among the constructions proposed in the literature (see Roy [2], Llamazares [3, 4], and Beliakov [5] for an analysis of some of them), those based on the Choquet integral are of special interest due mainly to two reasons. The first one is that both the weighted means and the OWA operators are specific cases of the Choquet integral. The second one is that Choquet integrals satisfy some desirable properties in certain information aggregation contexts: monotonicity, compensativeness, idempotency, continuity and homogeneity of degree 1.

One of the most interesting generalizations is the family of semiuninorm-based ordered weighted averaging (SUOWA) operators (Llamazares [6]). In these functions, the capacities on which Choquet integrals are based are

\footnotetext{
${ }^{*}$ Tel.: +34-983-186544; fax: +34-983-423299.

Email address: boni@eco.uva.es (Bonifacio Llamazares)
} 
built by using the capacities associated with the weighted means and the OWA operators, and "assembling" these values through a semiuninorm with neutral element $1 / n .{ }^{1}$ In addition to this natural construction, SUOWA operators have the following advantages over other approaches proposed in the literature:

1. Some indices such as the orness degree (Yager [1], Marichal [7]), the Shapley value (Shapley [8]), the veto and favor indices (Marichal [7]), and the $k$-conjunctiveness and $k$-disjunctiveness indices (Marichal [9]), which are of special interest in the study of games and capacities, can be given through closed-form expressions for some specific cases of SUOWA operators (Llamazares [10, 11, 12, 4, 13, 14]).

2. SUOWA operators allow us to preserve some of the main features of weighted means and OWA operators. Recall that weighted means allow to weight each information source in relation to their importance while OWA operators, which are in fact convex combinations of order statistics, allow that extreme values (also known as outliers) can be discarded by using appropriate weighting vectors (trimmed and Winsorized means are wellknown examples of OWA operators that possess this property). In this sense, SUOWA operators allow to get operators located between two order statistics that take into account the weights of the information sources (Llamazares $[12,4,13])^{2}$

It is also worth emphasizing that some families of operators recently introduced in the literature are closely related to SUOWA operators. For instance:

1. The Winsorized weighted mean introduced by Llamazares [13], which is the natural generalization of the Winsorized mean (see Dixon [15], and Wainer [16]), is a specific case of SUOWA operators.

2. The solution of a convex optimization problem proposed by Labreuche [17] is also a specific case of SUOWA operators (Llamazares $[18,11,12])$.

3. The games proposed by Jin et al. [19] in the Crescent Method can be expressed as a two-piecewise function where the first piece coincides with a game associated with a SUOWA operator and the second piece is the dual of a game also obtained in the context of SUOWA operators (see Llamazares [14]).

4. The games associated with Semi-SUOWA operators, which are generalizations of those used in the Crescent Method, were introduced by Llamazares [20] and are defined through a two-piecewise function where the first piece is a game associated with a SUOWA operator, $v_{p, w}^{U}$, and the second piece is the dual of the game obtained by using the same semiuninorm $U$, the same weighting vector $\boldsymbol{p}$, and the dual of $\boldsymbol{w}$, i.e., $\bar{v}_{\boldsymbol{p}, \overline{\boldsymbol{w}}}^{U}$.

The main aim of this paper is to analyze the games associated with Semi-SUOWA operators from different perspectives. Hence, we introduce a condition on the semiuninorm $U$ that allows us to obtain capacities when the weighting vector $\boldsymbol{w}$ is unimodal. It is worth noting that this result generalizes another one obtained by Llamazares [20] for a specific family of semiuninorms. In addition to this, we establish some relationships between some games

\footnotetext{
${ }^{1}$ In some cases the resulting game is not monotonic and it is necessary to consider the monotonic cover of the game to obtain a capacity.

${ }^{2}$ Notice that this property is not fulfilled for other generalizations proposed in the literature (see Beliakov [5]).
} 
associated with SUOWA and Semi-SUOWA operators and, in the case of nondecreasing and nonincreasing weighting vectors $\boldsymbol{w}$, we give simple Hasse diagrams showing the usual partial order between these games. Lastly, we also show the pointwise convergence of certain games.

The remainder of the paper is organized as follows. In Section 2 we recall some concepts and results in the framework of SUOWA and Semi-SUOWA operators. In Section 3 we give conditions that guarantee the monotonicity of the games associated with Semi-SUOWA operators. Moreover, we also establish some relationships between some games associated with SUOWA and Semi-SUOWA operators. Section 4 is devoted to show the pointwise convergence of some games. Finally, some concluding remarks are provided in Section 5.

\section{Preliminaries}

Throughout the paper we will use the following notation: $N$ denotes the set $\{1, \ldots, n\},|A|$ and $A^{\mathrm{c}}$ denote, respectively, the cardinality and the complement of a subset $A$ of $N$, vectors are denoted in bold, $\boldsymbol{\eta}$ is the vector $(1 / n, \ldots, 1 / n) \in \mathbb{R}^{n}$, and given $\boldsymbol{x} \in \mathbb{R}^{n},[\cdot]$ and $(\cdot)$ denote permutations such that $x_{[1]} \geq \cdots \geq x_{[n]}$ and $x_{(1)} \leq \cdots \leq x_{(n)}$.

A game $v$ on $N$ is a set function, $v: 2^{N} \longrightarrow \mathbb{R}$ satisfying $v(\varnothing)=0$. Monotonic games are called capacities, and a capacity $\mu$ is normalized if $\mu(N)=1$ (see Mesiar et al. [21] for an interesting study on the constructions of normalized capacities by means of aggregation and implication functions). The monotonic cover (see Maschler and Peleg [22], and Maschler et al. [23]) of a game $v$ is the set function $\hat{v}$ defined by

$$
\widehat{v}(A)=\max _{B \subseteq A} v(B)
$$

By construction, $\hat{v}$ is a capacity, and $\hat{v}=v$ when $v$ is a capacity. Moreover, $\widehat{v}$ is a normalized capacity when $v(N)=1$ and $v(A) \leq 1$ for all $A \subseteq N$. The dual of a game $v$ is the game defined by $\bar{v}(A)=1-v\left(A^{\mathrm{c}}\right)$, where $A \subseteq N$. It is easy to check that the dual of a normalized capacity is also a normalized capacity.

The Choquet integral (see, for instance, Choquet [24], Denneberg [25], and Grabisch et al. [26]) with respect to a normalized capacity $\mu$ is the function $C_{\mu}: \mathbb{R}^{n} \longrightarrow \mathbb{R}$ given by

$$
C_{\mu}(\boldsymbol{x})=\sum_{i=1}^{n} \mu\left(A_{[i]}\right)\left(x_{[i]}-x_{[i+1]}\right),
$$

where $A_{[i]}=\{[1], \ldots,[i]\}$, and we use the convention $x_{[n+1]}=0$.

Weighted means and OWA operators are two of the best-known specific cases of Choquet integrals. Both are defined through weighting vectors; that is, nonnegative vectors whose components sum to $1 .^{3}$ The weighted mean $M_{p}$ associated with a weighting vector $\boldsymbol{p}$ is the Choquet integral with respect to the normalized capacity $\mu_{p}(A)=\sum_{i \in A} p_{i}$, that is,

$$
M_{p}(\boldsymbol{x})=\sum_{i=1}^{n} p_{i} x_{i}
$$

\footnotetext{
${ }^{3}$ It is worth noting that the choice of the weight distribution has generated a large literature (in the case of OWA operators, see, for instance, Llamazares [27] and Liu [28]).
} 
whereas the OWA operator $O_{w}$ associated with a weighting vector $w$ is the Choquet integral with respect to the normalized capacity $\mu_{|w|}(A)=\sum_{i=1}^{|A|} w_{i}$, that is,

$$
O_{w}(\boldsymbol{x})=\sum_{i=1}^{n} w_{i} x_{[i]} .
$$

Notice that OWA operators are convex combinations of order statistics. Moreover, the dual of $\mu_{|w|}$ (that for the sake of simplicity we will denote by $\bar{\mu}_{|\boldsymbol{w}|}$ instead of $\left.\overline{\mu_{\mid \boldsymbol{w}} \mid}\right)$ is given by $\mu_{|\bar{w}|}$, where $\overline{\boldsymbol{w}}$ is the dual of $\boldsymbol{w}$; that is, $\overline{\boldsymbol{w}}=\left(w_{n}, w_{n-1}, \ldots, w_{1}\right)$ (equivalently, $\bar{w}_{i}=w_{n+1-i}$ ).

An outstanding family of weighting vectors are the unimodal ones (see Llamazares [29]). A weighting vector $w$ is unimodal if there exists an index $k$ such that $w_{1} \leq \cdots \leq w_{k-1} \leq w_{k} \geq w_{k+1} \geq \cdots \geq w_{n}$. Notice that unimodal weighting vectors embrace, among others, nondecreasing $\left(w_{1} \leq \cdots \leq w_{n}\right)$, nonincreasing $\left(w_{1} \geq \cdots \geq w_{n}\right)$, and centered weighting vectors (Yager [30]). The set of unimodal weighting vectors will be denoted by $\mathcal{W}_{\mathrm{u}}$.

The following lemmas, which gather some properties on unimodal weighting vectors, have been proven by Llamazares $[29,20]$.

Lemma 1. Given $\boldsymbol{w} \in \mathcal{W}_{\mathrm{u}}$, if we define

$$
\begin{array}{lll}
L_{w}=\left\{l \in N \mid \sum_{i=1}^{l} w_{i}<\frac{l}{n}\right\}, & L^{w}=\left\{l \in N \mid \sum_{i=1}^{l} w_{i}>\frac{l}{n}\right\}, \\
l_{w}= \begin{cases}0, & \text { if } L_{w}=\varnothing, \\
\max L_{w}, & \text { otherwise, }\end{cases} & l^{w}= \begin{cases}n+1, & \text { if } L^{w}=\varnothing, \\
\min L^{w}, & \text { otherwise, }\end{cases}
\end{array}
$$

then

1. $l_{w}<l^{w}$.

2. If $L_{w} \neq \varnothing$, then $L_{w}=\left\{1, \ldots, l_{w}\right\}$.

3. If $L^{w} \neq \varnothing$, then $L^{w}=\left\{l^{w}, \ldots, n-1\right\}$.

According to the previous lemma, each unimodal weighting vector $w$ has associated two indices, $l_{w}$ and $l^{w}$, so given $\varnothing \neq A \varsubsetneqq N$ we have

1. $|A| \leq l_{w} \Leftrightarrow \mu_{|w|}(A) /|A|<1 / n$.

2. $l_{w}<|A|<l^{w} \Leftrightarrow \mu_{|w|}(A) /|A|=1 / n$.

3. $l^{w} \leq|A| \Leftrightarrow \mu_{|w|}(A) /|A|>1 / n$.

The next lemma shows some relationships between the indices $l_{w}$ and $l^{w}$ of a unimodal weighting vector and its dual.

Lemma 2. Let $w \in \mathcal{W}_{\mathrm{u}}$. Then

1. $l_{w}=0 \Leftrightarrow l^{\bar{w}}=n+1$. 
2. If $l_{w} \neq 0$, then $l_{w}+l^{\bar{w}}=n$.

3. $l^{w}=n+1 \Leftrightarrow l_{\bar{w}}=0$.

4. If $l^{w} \neq n+1$, then $l^{w}+l_{\bar{w}}=n$.

The last lemma establishes that the sequence formed by the averages of the components of a unimodal weighting vector $\boldsymbol{w}$ is nondecreasing up to the index $l_{w}$.

Lemma 3. Let $\boldsymbol{w} \in \mathcal{W}_{\mathrm{u}}$ and $p, q \in N$ with $p<q \leq l_{w}$. Then

$$
\frac{1}{p} \sum_{i=1}^{p} w_{i} \leq \frac{1}{q} \sum_{i=1}^{q} w_{i} .
$$

In the next subsections we recall some fundamental notions on semiuninorms, SUOWA, and Semi-SUOWA operators.

\subsection{Semiuninorms}

SUOWA operators were introduced by Llamazares [6] as a generalization of weighted means and OWA operators. In their definition, semiuninorms (see Liu [31]) play a fundamental role. A semiuninorm is a monotonic function $U:[0,1]^{2} \longrightarrow[0,1]$ that has a neutral element $e .^{4}$ The structure of semiuninorms has been studied by Liu [31, Propositions 2.1 and 2.2] and it is showed in Figure 1.

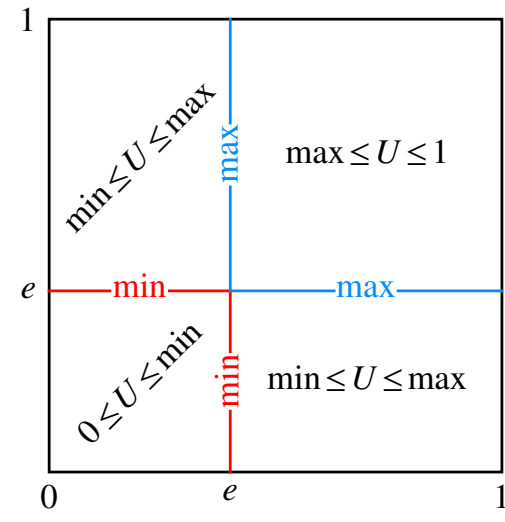

(a) Generic semiuninorms

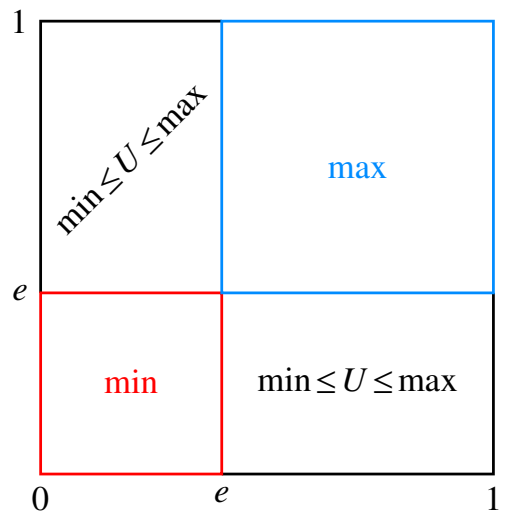

(b) Idempotent semiuninorms.

Figure 1: The structure of semiuninorms.

The set of semiuninorms with $1 / n$ as neutral element is denoted by $\mathcal{U}^{1 / n}$, and the semiuninorms used in the definition of SUOWA operators have to belong to the following subset (see Llamazares [6]):

$$
\widetilde{\mathcal{U}}^{1 / n}=\left\{U \in \mathcal{U}^{1 / n} \mid U(1 / k, 1 / k) \leq 1 / k \text { for all } k \in N\right\}
$$

\footnotetext{
${ }^{4}$ Semiuninorms are, in fact, generalizations of uninorms (Yager and Rybalov [32]) where the commutativity and associativity properties are ruled out.
} 
In Table 1 we collect some relevant semiuninorms that have been analyzed in the literature $[33,34,4]$. Notice that $U_{\perp}$ and $U_{\mathrm{T}}$ are, respectively, the smallest and the largest elements of $\widetilde{\mathcal{U}}^{1 / n}$, whereas $U_{\min }$ and $U_{\max }$ are, respectively, the smallest and the largest idempotent semiuninorms of $\widetilde{\mathcal{U}}^{1 / n}$. A Hasse diagram showing the usual partial order between the semiuninorms given in Table 1 can be found in Llamazares [4].

In addition to the previous ones, a new family of semiuninorms has been introduced in a recent paper (see Llamazares [20]) by using strictly increasing functions: If $h:[0,1] \longrightarrow[-\infty, \infty]$ is a strictly increasing function with $\{-\infty, \infty\} \nsubseteq \operatorname{Ran}(h)$, the semiuninorm $U_{h}^{\max }$ is defined by

$$
U_{h}^{\max }(x, y)= \begin{cases}H(x, y) & \text { if } y \leq 1 / n, \\ \max (x, y) & \text { if } y>1 / n,\end{cases}
$$

where $H(x, y)=h^{(-1)}(h(x)+h(y)-h(1 / n))$ and $h^{(-1)}$ is the pseudo-inverse of $h$ (see Klement et al. [35]). Notice that $U_{h}^{\max }$ is continuous when $h$ is continuous.

\subsection{SUOWA operators}

As we have seen previously, capacities play a fundamental role in the definition of Choquet integrals. In the case of SUOWA operators, the capacities are the monotonic cover of certain games, which are defined by using semiuninorms with neutral element $1 / n$ and the values of the capacities associated with the weighted means and the OWA operators.

Definition 1. Let $\boldsymbol{p}$ and $\boldsymbol{w}$ be two weighting vectors and let $U \in \widetilde{\mathcal{U}}^{1 / n}$.

1. The game $v_{p, w}^{U}$ associated with $\boldsymbol{p}, \boldsymbol{w}$ and $U$ is the set function defined by

$$
v_{p, w}^{U}(A)=|A| U\left(\frac{\mu_{p}(A)}{|A|}, \frac{\mu_{|w|}(A)}{|A|}\right)=|A| U\left(\frac{\sum_{i \in A} p_{i}}{|A|}, \frac{\sum_{i=1}^{|A|} w_{i}}{|A|}\right),
$$

if $A \neq \varnothing$, and $v_{p, w}^{U}(\varnothing)=0$.

2. The SUOWA operator associated with $\boldsymbol{p}, \boldsymbol{w}$ and $U$ is the Choquet integral with respect to the capacity $\widehat{v}_{\boldsymbol{p}, \boldsymbol{w}}^{U}$ (that is, the monotonic cover of $v_{p, w}^{U}$, and it will be denoted by $S_{p, w}^{U}$.

Note that, by construction, SUOWA operators allow us to recover the weighted mean $M_{p}$ when $\boldsymbol{w}=\boldsymbol{\eta}$ and the OWA operator $O_{\boldsymbol{w}}$ when $\boldsymbol{p}=\boldsymbol{\eta}$; that is, $\hat{v}_{\boldsymbol{p}, \boldsymbol{\eta}}^{U}=\mu_{\boldsymbol{p}}$ and $\hat{v}_{\boldsymbol{\eta}, \boldsymbol{w}}^{U}=\mu_{|\boldsymbol{w}|}$ for any $U \in \widetilde{\mathcal{U}}^{1 / n}$. A summary of the main properties of SUOWA operators can be found in Llamazares [4].

In the case of the semiuninorm $U_{h}^{\max }$, the game $v_{p, w}^{U_{h}^{\max }}$, which for simplicity will be denoted by $v_{p, w}^{h}$, is given by

$$
v_{p, w}^{h}(A)= \begin{cases}H_{p, w}(A) & \text { if } \mu_{|w|}(A) \leq|A| / n, \\ \max \left(\mu_{p}(A), \mu_{|w|}(A)\right) & \text { if } \mu_{|w|}(A)>|A| / n,\end{cases}
$$

where $A$ is any nonempty subset of $N$ and

$$
H_{p, w}(A)=|A| H\left(\frac{\mu_{p}(A)}{|A|}, \frac{\mu_{|w|}(A)}{|A|}\right)=|A| h^{(-1)}\left(h\left(\frac{\mu_{p}(A)}{|A|}\right)+h\left(\frac{\mu_{|w|}(A)}{|A|}\right)-h(1 / n)\right) .
$$


Table 1: Some relevant semiuninorms and their structure.

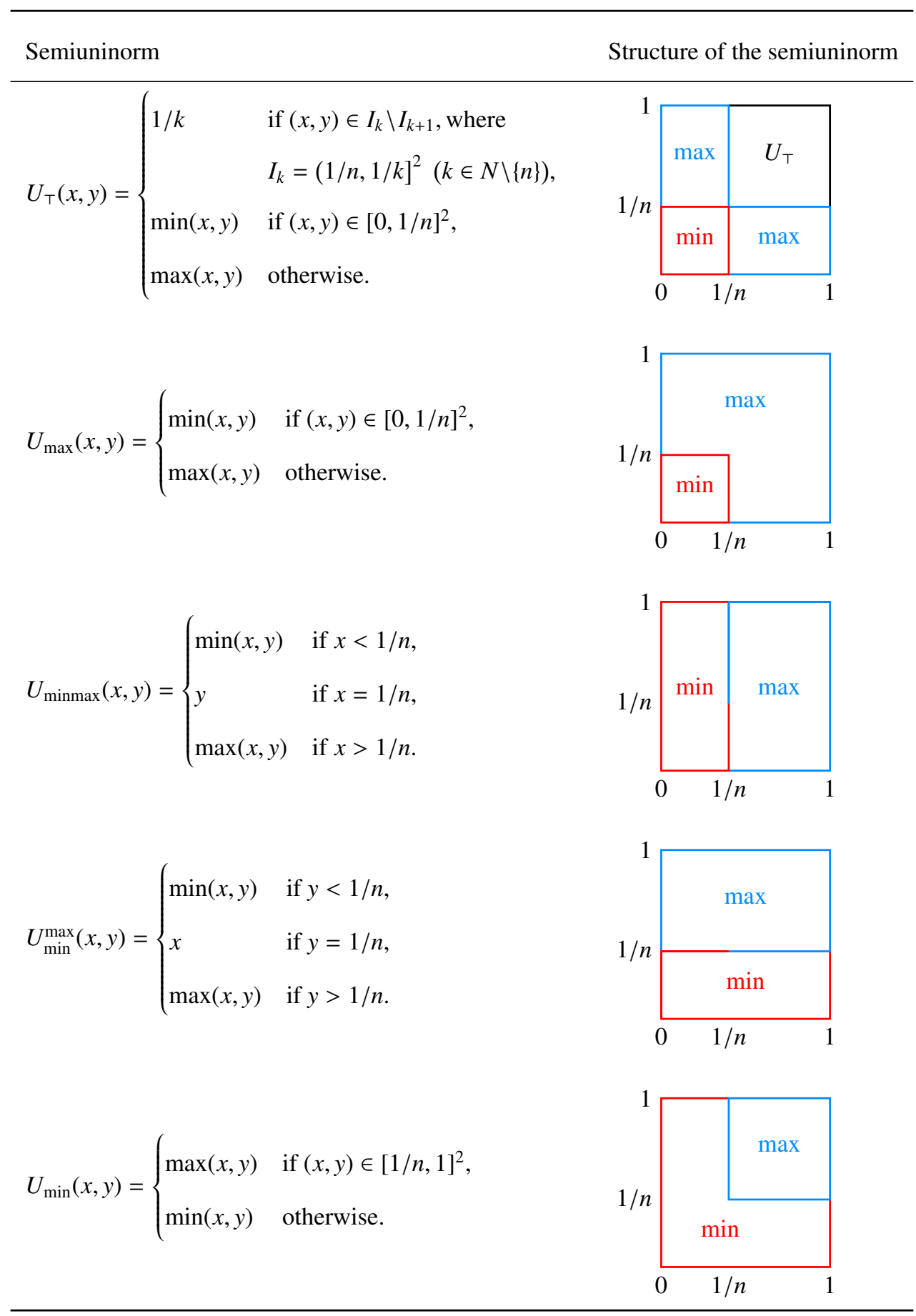

Remark 1. It is worth noting that the partial order between semiuninorms is preserved by the games, the capacities, and the SUOWA operators; that is, if $\boldsymbol{p}$ and $\boldsymbol{w}$ are two weighting vectors, and $U_{1}, U_{2} \in \widetilde{\mathcal{U}}^{1 / n}$, with $U_{1} \leq U_{2}$, then 


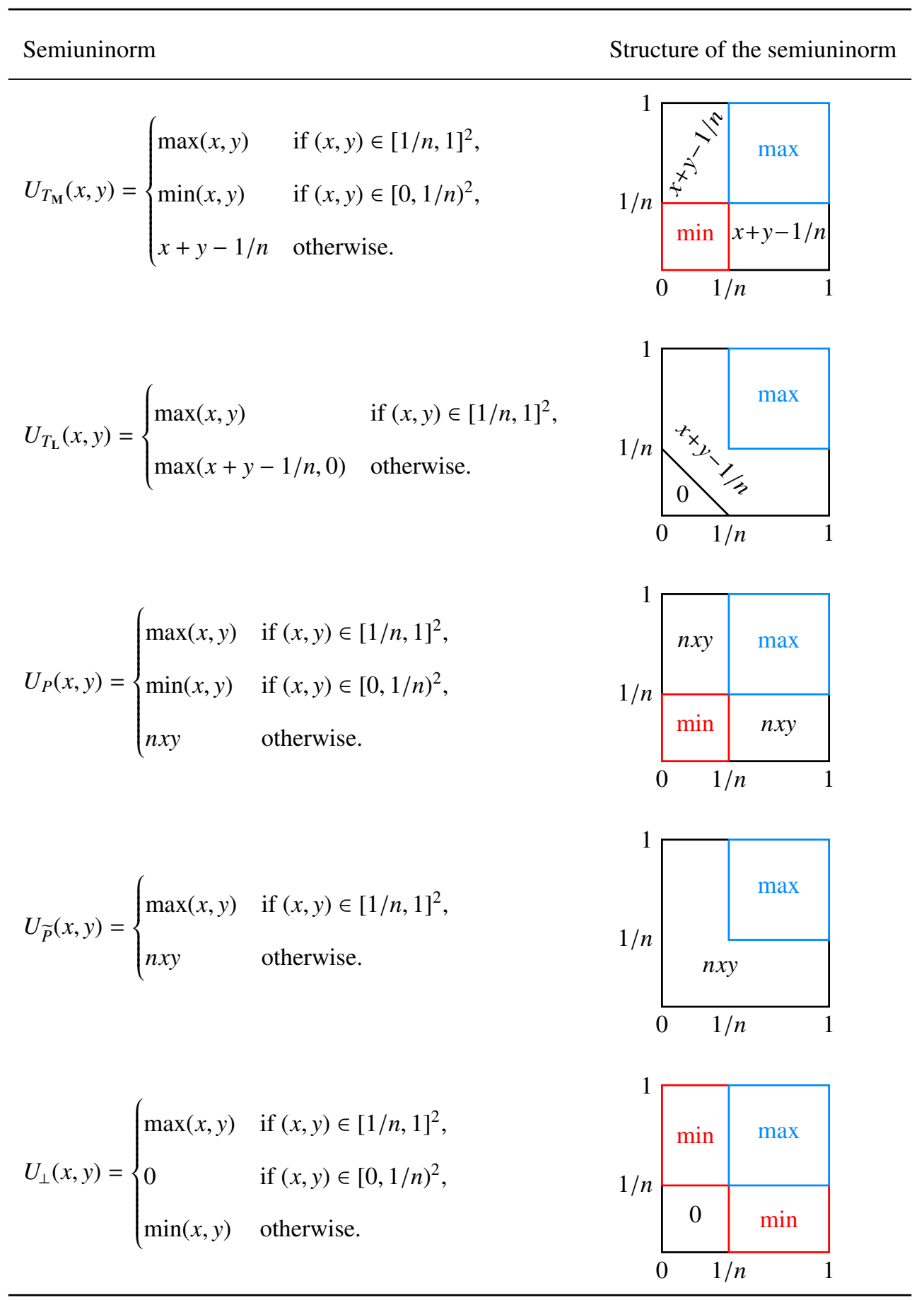

$v_{\boldsymbol{p}, \boldsymbol{w}}^{U_{1}} \leq v_{\boldsymbol{p}, \boldsymbol{w}}^{U_{2}}, \hat{v}_{\boldsymbol{p}, \boldsymbol{w}}^{U_{1}} \leq \hat{v}_{\boldsymbol{p}, \boldsymbol{w}}^{U_{2}}$, and $S_{\boldsymbol{p}, \boldsymbol{w}}^{U_{1}} \leq S_{\boldsymbol{p}, \boldsymbol{w}}^{U_{2}}$. Hence, a Hasse diagram similar to that shown by Llamazares [4] for some semiuninorms can be performed for the corresponding games, capacities and SUOWA operators. Notice also that if 
$U \in \widetilde{\mathcal{U}}^{1 / n}$, then $v_{\boldsymbol{p}, \boldsymbol{w}}^{U_{\perp}} \leq v_{\boldsymbol{p}, \boldsymbol{w}}^{U} \leq v_{\boldsymbol{p}, \boldsymbol{w}}^{U_{\top}}, \hat{v}_{\boldsymbol{p}, \boldsymbol{w}}^{U_{\perp}} \leq \hat{v}_{\boldsymbol{p}, \boldsymbol{w}}^{U} \leq \hat{v}_{\boldsymbol{p}, \boldsymbol{w}}^{U_{\top}}$, and $S_{\boldsymbol{p}, \boldsymbol{w}}^{U_{\perp}} \leq S_{\boldsymbol{p}, \boldsymbol{w}}^{U} \leq S_{\boldsymbol{p}, \boldsymbol{w}}^{U_{\top}}$

\subsection{Semi-SUOWA operators}

The games associated with this family of operators are closely related to those associated with SUOWA operators. In fact, they coincide with $v_{p, w}^{U}(A)$ when $\mu_{|w|}(A) \leq|A| / n$, and with $\bar{v}_{p, \bar{w}}^{U}(A)$ when $\mu_{|\boldsymbol{w}|}(A)>|A| / n$ (notice that $\bar{v}_{\boldsymbol{p}, \bar{w}}^{U}$ is the dual of the game associated with $p, \bar{w}$ and $U$ ).

Definition 2. Let $\boldsymbol{p}$ and $\boldsymbol{w}$ be two weighting vectors and let $U \in \widetilde{\mathcal{U}}^{1 / n}$.

1. The game $\xi_{p, w}^{U}$ associated with $\boldsymbol{p}, \boldsymbol{w}$ and $U$ is the set function defined by

$$
\begin{aligned}
\xi_{\boldsymbol{p}, \boldsymbol{w}}^{U}(A) & = \begin{cases}v_{\boldsymbol{p}, \boldsymbol{w}}^{U}(A) & \text { if } \mu_{|\boldsymbol{w}|}(A) \leq|A| / n, \\
\bar{v}_{\boldsymbol{p}, \overline{\boldsymbol{w}}}^{U}(A)=\bar{\xi}_{\boldsymbol{p}, \bar{w}}^{U}(A)=1-\xi_{\boldsymbol{p}, \bar{w}}^{U}\left(A^{\mathrm{c}}\right) & \text { if } \mu_{|\boldsymbol{w}|}(A)>|A| / n,\end{cases} \\
& = \begin{cases}|A| U\left(\frac{\mu_{p}(A)}{|A|}, \frac{\mu_{|w|}(A)}{|A|}\right) & \text { if } \mu_{|\boldsymbol{w}|}(A) \leq|A| / n, \\
1-(n-|A|) U\left(\frac{1-\mu_{p}(A)}{n-|A|}, \frac{1-\mu_{|\boldsymbol{w}|}(A)}{n-|A|}\right) & \text { if } \mu_{|\boldsymbol{w}|}(A)>|A| / n,\end{cases}
\end{aligned}
$$

if $A \neq \varnothing$, and $\xi_{p, w}^{U}(\varnothing)=0$.

2. The Semi-SUOWA operator associated with $\boldsymbol{p}, \boldsymbol{w}$ and $U$ is the Choquet integral with respect to the capacity $\hat{\xi}_{\boldsymbol{p}, \boldsymbol{w}}^{U}$ (that is, the monotonic cover of $\xi_{p, w}^{U}$ ).

It is worthy of noting that if $\mu_{|w|}(A) \leq|A| / n$ then $\mu_{|w|}(A) /|A| \leq 1 / n$, and when $\mu_{|w|}(A)>|A| / n$ we also have $\left(1-\mu_{|w|}(A)\right) /(n-|A|)<1 / n$. Therefore the games $\xi_{p, w}^{U}$ only use the values of $U$ in the region $[0,1] \times[0,1 / n]$; hence, instead of semiuninorms we can consider in their definition any monotonic function $U:[0,1] \times[0,1 / n] \longrightarrow[0,1]$ such that $U(1 / n, y)=y$ and $U(x, 1 / n)=x$.

Notice also that when $\mu_{|w|}(A)=|A| / n$, we have $\xi_{p, w}^{U}(A)=\mu_{p}(A)$. Since this value is also obtained with the expression $1-(n-|A|) U\left(\frac{1-\mu_{p}(A)}{n-|A|}, \frac{1-\mu_{|w|}(A)}{n-|A|}\right)$, when $A \neq \varnothing$ the game $\xi_{p, w}^{U}$ can also be written as

$$
\xi_{p, w}^{U}(A)= \begin{cases}|A| U\left(\frac{\mu_{p}(A)}{|A|}, \frac{\mu_{|w|}(A)}{|A|}\right) & \text { if } \mu_{|w|}(A)<|A| / n \\ 1-(n-|A|) U\left(\frac{1-\mu_{p}(A)}{n-|A|}, \frac{1-\mu_{|w|}(A)}{n-|A|}\right) & \text { if } \mu_{|w|}(A) \geq|A| / n\end{cases}
$$

As in the case of SUOWA operators, Semi-SUOWA operators allow us to recover the weighted mean $M_{p}$ when $\boldsymbol{w}=\boldsymbol{\eta}$ and the OWA operator $O_{\boldsymbol{w}}$ when $\boldsymbol{p}=\boldsymbol{\eta}$; that is, $\hat{\xi}_{\boldsymbol{p}, \boldsymbol{\eta}}^{U}=\mu_{\boldsymbol{p}}$ and $\hat{\xi}_{\boldsymbol{\eta}, \boldsymbol{w}}^{U}=\mu_{|\boldsymbol{w}|}$ for any $U \in \widetilde{\mathcal{U}}^{1 / n}$.

In the case of the semiuninorm $U_{h}^{\max }$, the game $\xi_{p, \boldsymbol{w}}^{U_{h}^{\max }}$, which for simplicity will be denoted by $\xi_{\boldsymbol{p}, \boldsymbol{w}}^{h}$, is given by

$$
\xi_{p, w}^{h}(A)= \begin{cases}H_{p, w}(A), & \text { if } \mu_{|w|}(A) \leq|A| / n, \\ 1-H_{p, \bar{w}}\left(A^{\mathrm{c}}\right), & \text { if } \mu_{|w|}(A)>|A| / n,\end{cases}
$$


that is,

$$
\xi_{p, w}^{h}(A)= \begin{cases}|A| h^{(-1)}\left(h\left(\frac{\mu_{p}(A)}{|A|}\right)+h\left(\frac{\mu_{|w|}(A)}{|A|}\right)-h(1 / n)\right) & \text { if } \mu_{|w|}(A) \leq|A| / n, \\ 1-(n-|A|) h^{(-1)}\left(h\left(\frac{1-\mu_{p}(A)}{n-|A|}\right)+h\left(\frac{1-\mu_{|w|}(A)}{n-|A|}\right)-h(1 / n)\right) & \text { if } \mu_{|w|}(A)>|A| / n,\end{cases}
$$

where $A$ is any nonempty subset of $N$.

We finish this section by showing that the games associated with SUOWA and Semi-SUOWA operators are different families of games (and none of them is contained in the other). To do this, consider the weighting vectors $\boldsymbol{p}=(0.5,0.2,0.2,0.1)$ and $\boldsymbol{w}=(0.4,0.1,0.1,0.4)$. It is easy to check that $\xi_{p, w}^{U_{\perp}}(\{1\})=1$ whereas $v_{p, w}^{U_{\top}}(\{1\})=0.5$. Therefore, according to Remark 1 , there is no semiuninorm $U \in \widetilde{\mathcal{U}}^{1 / n}$ such that $v_{p, w}^{U}=\xi_{p, w}^{U_{\perp}}$. Reciprocally, suppose there exists a semiuninorm $U \in \widetilde{\mathcal{U}}^{1 / n}$ such that $\xi_{\boldsymbol{p}, \boldsymbol{w}}^{U}=v_{\boldsymbol{p}, \boldsymbol{w}}^{U_{\perp}}$. Then $3 U(0.5 / 3,0.2)=\xi_{\boldsymbol{p}, \boldsymbol{w}}^{U}(\{2,3,4\})=v_{\boldsymbol{p}, \boldsymbol{w}}^{U_{\perp}}(\{2,3,4\})=0$. But then $\xi_{p, w}^{U}(\{1\})=1-3 U(0.5 / 3,0.2)=1 \neq 0.5=v_{p, w}^{U_{\perp}}(\{1\})$, which contradicts the hypothesis.

\section{Some results on the games $\xi_{p, w}^{U}$}

In this section we analyze some games associated with Semi-SUOWA operators. On the one hand, we introduce a condition on the semiuninorm $U$ that allows us to obtain capacities when the weighting vector $w$ is unimodal. On the other hand, we establish some relationships between the games $\xi_{p, w}^{U}$ and $v_{p, w}^{U}$ for the semiuninorms given in Table 1 . Of special interest are the cases in which the weighting vector $w$ is nondecreasing or nonincreasing because we can provide simple Hasse diagrams showing the usual partial order between these games.

\subsection{Obtaining capacities with unimodal weighting vectors}

In this subsection we establish conditions under which we can assure that the game $\xi_{p, w}^{U}$ is a capacity. To be specific, we give a condition on the semiuninorm $U$ that guarantees the monotonicity of the game $\xi_{p, w}^{U}$ when $w \in \mathcal{W}_{\mathrm{u}}$. To this end, we will use the following lemma.

Lemma 4. Let $\boldsymbol{w} \in \mathcal{W}_{\mathrm{u}}$ and let $U$ be a semiuninorm such that $U(t x, y) \leq t U(x, y)$ for any $y \leq 1 / n, x \in[0,1]$, and $t>1$ such that $t x \in[0,1]$. Then, for any weighting vector $p, \xi_{p, w}^{U}(A) \leq \xi_{p, w}^{U}(B)$ for any $A, B \subseteq N$, with $\varnothing \neq A \subsetneq B$ and $|B| \leq l_{w}$.

Proof. Let $\boldsymbol{p}$ and $\boldsymbol{w}$ be two weighting vectors with $\boldsymbol{w} \in \mathcal{W}_{\mathrm{u}}$, and let $U$ be a semiuninorm satisfying the hypotheses. If $\varnothing \neq A \subsetneq B$ and $|B| \leq l_{w}$, then, by Lemma 3 we have $\mu_{|w|}(A) /|A| \leq \mu_{|w|}(B) /|B|$. Therefore,

$$
\xi_{p, w}^{U}(A)=|A| U\left(\frac{\mu_{p}(A)}{|A|}, \frac{\mu_{|w|}(A)}{|A|}\right) \leq|A| U\left(\frac{|B|}{|A|} \frac{\mu_{p}(B)}{|B|}, \frac{\mu_{|w|}(B)}{|B|}\right) \leq|B| U\left(\frac{\mu_{p}(B)}{|B|}, \frac{\mu_{|w|}(B)}{|B|}\right)=\xi_{p, w}^{U}(B) .
$$

Theorem 1. Let $w \in \mathcal{W}_{\mathrm{u}}$ and let $U$ be a semiuninorm such that $U(t x, y) \leq t U(x, y)$ for any $y \leq 1 / n, x \in[0,1]$, and $t>1$ such that $t x \in[0,1]$. Then, for any weighting vector $\boldsymbol{p}, \xi_{\boldsymbol{p}, \boldsymbol{w}}^{U}$ is a normalized capacity on $N$. 
Proof. Let $\boldsymbol{p}$ and $\boldsymbol{w}$ be two weighting vectors with $\boldsymbol{w} \in \mathcal{W}_{\mathrm{u}}$. Consider $A \subsetneq B$ with $|A| \geq 1$ and $|B|<n$ (the cases $A=\varnothing$ and $B=N$ are trivial). We distinguish the following cases:

1. If $|B| \leq l_{w}$, then by Lemma $4, \xi_{p, w}^{U}(A) \leq \xi_{p, w}^{U}(B)$.

2. If $|A| \leq l_{w}$ and $l_{w}<|B|<l^{w}$, then

$$
\xi_{p, w}^{U}(A) \leq|A| U\left(\frac{\mu_{p}(A)}{|A|}, \frac{1}{n}\right)=\mu_{p}(A) \leq \mu_{p}(B)=|B| U\left(\frac{\mu_{p}(B)}{|B|}, \frac{1}{n}\right)=\xi_{p, w}^{U}(B) .
$$

3. If $l_{w}<|A|<|B|<l^{w}$, then $\xi_{p, w}^{U}(A)=\mu_{p}(A) \leq \mu_{p}(B)=\xi_{p, w}^{U}(B)$.

4. If $|A|<l^{w}$ and $|B| \geq l^{w}$, then by Lemma $2,\left|B^{\complement}\right|=n-|B| \leq n-l^{w}=l_{\bar{w}}$. So,

$$
\xi_{p, w}^{U}(A) \leq \mu_{p}(A) \leq \mu_{p}(B)=1-\mu_{p}\left(B^{\mathrm{C}}\right) \leq 1-\xi_{p, \bar{w}}^{U}\left(B^{\mathrm{C}}\right)=\xi_{p, w}^{U}(B)
$$

5. If $|B|>|A| \geq l^{w}$, then by Lemma $2,\left|B^{\mathrm{c}}\right|<\left|A^{\mathrm{c}}\right| \leq l_{\bar{w}}$; and, by Lemma $4, \xi_{p, \bar{w}}^{U}\left(B^{\mathrm{c}}\right) \leq \xi_{p, \bar{w}}^{U}\left(A^{\mathrm{c}}\right)$. Therefore,

$$
\xi_{\boldsymbol{p}, \boldsymbol{w}}^{U}(A)=1-\xi_{\boldsymbol{p}, \overline{\boldsymbol{w}}}^{U}\left(A^{\mathrm{c}}\right) \leq 1-\xi_{\boldsymbol{p}, \bar{w}}^{U}\left(B^{\mathrm{C}}\right)=\xi_{\boldsymbol{p}, \boldsymbol{w}}^{U}(B)
$$

It is obvious to check that the semiuninorm $U_{\widetilde{P}}$ satisfies the condition given in Theorem 1 . Therefore, the game $\xi_{\boldsymbol{p}, \boldsymbol{w}}^{U_{\widetilde{w}}}$ is a capacity when $\boldsymbol{w}$ is unimodal. ${ }^{5}$ Next we show that also the semiuninorm $U_{P}$ satisfies the condition given in Theorem 1 and, consequently, $\xi_{p, w}^{U_{P}}$ is a capacity when $\boldsymbol{w} \in \mathcal{W}_{\mathrm{u}}$.

Proposition 1. The semiuninorm $U_{P}$ satisfies that $U_{P}(t x, y) \leq t U_{P}(x, y)$ for any $y \leq 1 / n, x \in[0,1]$, and $t>1$ such that $t x \in[0,1]$.

Proof. Consider $y \leq 1 / n, x \in[0,1]$, and $t>1$ such that $t x \in[0,1]$. We distinguish the following cases:

1. If $t x \leq y$, then $U_{P}(t x, y)=\min (t x, y)=t x=t \min (x, y)=t U_{P}(x, y)$.

2. If $y<t x \leq 1 / n$, then $U_{P}(t x, y)=\min (t x, y)=y<\min (t x, t y)=t \min (x, y)=t U_{P}(x, y)$.

3. If $1 / n<x$, then $U_{P}(t x, y)=n t x y=t U_{P}(x, y)$.

4. If $x \leq 1 / n<t x$, then $U_{P}(t x, y)=n t x y=t n \max (x, y) \min (x, y) \leq t \min (x, y)=t U_{P}(x, y)$.

Corollary 1. Let $\boldsymbol{w} \in \mathcal{W}_{\mathrm{u}}$. Then, for any weighting vector $\boldsymbol{p}, \xi_{p, w}^{U_{P}}$ is a normalized capacity on $N$.

It is worth noting that Theorem 1 generalizes the result obtained by Llamazares [20] for the games $\xi_{p, w}^{h}$. A similar generalization can be given for the games $v_{p, w}^{h}$.

Theorem 2. Let $\boldsymbol{w} \in \mathcal{W}_{\mathrm{u}}$ and let $U$ be a semiuninorm such that $\left.U\right|_{[0,1] \times(1 / n, 1]}=\max$ and $U(t x, y) \leq t U(x, y)$ for any $y \leq 1 / n, x \in[0,1]$, and $t>1$ such that $t x \in[0,1]$. Then, for any weighting vector $\boldsymbol{p}, v_{\boldsymbol{p}, \boldsymbol{w}}^{U}$ is a normalized capacity on $N$.

\footnotetext{
${ }^{5}$ Notice that the game $\xi_{p, w}^{U_{\widetilde{P}}}$ coincides with the game obtained with the Crescent Method (see Llamazares [14]), and it has already been proven, using another method, that it is a capacity when $\boldsymbol{w}$ is unimodal (see Llamazares [20]).
} 


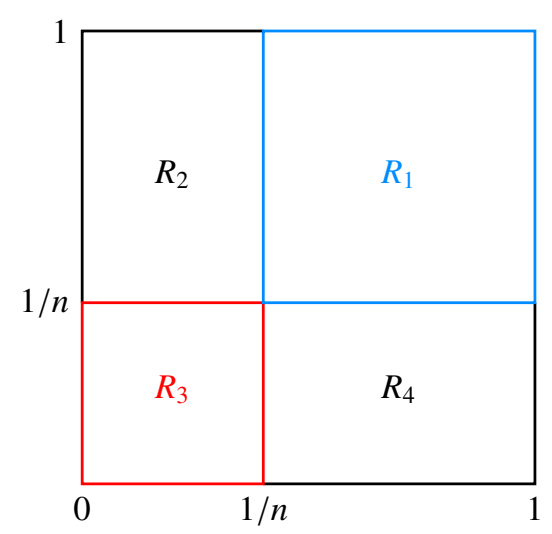

Figure 2: Regions of the square $[0,1]^{2}$.

Proof. Let $\boldsymbol{p}$ and $\boldsymbol{w}$ be two weighting vectors with $\boldsymbol{w} \in \mathcal{W}_{\mathrm{u}}$. Consider $A \subsetneq B$ with $|A| \geq 1$ and $|B|<n$ (the cases $A=\varnothing$ and $B=N$ are trivial). We distinguish the following cases:

1. If $|B| \leq l_{\boldsymbol{w}}$, then by Lemma $4, v_{\boldsymbol{p}, \boldsymbol{w}}^{U}(A)=\xi_{\boldsymbol{p}, \boldsymbol{w}}^{U}(A) \leq \xi_{\boldsymbol{p}, \boldsymbol{w}}^{U}(B)=v_{\boldsymbol{p}, \boldsymbol{w}}^{U}(B)$.

2. If $|B|>l_{w}$ and $|A|<l^{w}$, then $v_{p, w}^{U}(A) \leq \mu_{p}(A) \leq \mu_{p}(B) \leq v_{p, w}^{U}(B)$.

3. If $|A| \geq l^{w}$, then $v_{p, w}^{U}(A)=\max \left(\mu_{p}(A), \mu_{|w|}(A)\right) \leq \max \left(\mu_{p}(B), \mu_{|w|}(B)\right)=v_{p, w}^{U}(B)$.

\subsection{Some relationships between $\xi_{\boldsymbol{p}, \boldsymbol{w}}^{U}$ and $v_{\boldsymbol{p}, \boldsymbol{w}}^{U}$}

Notice that by definition of the games $\xi_{p, w}^{U}$, semiuninorms defined in the same way in the region $[0,1] \times[0,1 / n]$ return the same games $\xi_{\boldsymbol{p}, \boldsymbol{w}}^{U}$. Therefore, $\xi_{\boldsymbol{p}, \boldsymbol{w}}^{U_{\min }}=\xi_{\boldsymbol{p}, \boldsymbol{w}}^{U_{\min }^{\max }}$, and $\xi_{\boldsymbol{p}, \boldsymbol{w}}^{U_{\top}}=\xi_{\boldsymbol{p}, \boldsymbol{w}}^{U_{\max }}=\xi_{\boldsymbol{p}, \boldsymbol{w}}^{U_{\min m a x}}$ (see Table 1). In addition to this, we are going to prove that these games coincide with $v_{p, w}^{U_{\operatorname{mix}}^{\max }}$ and $v_{\boldsymbol{p}, \boldsymbol{w}}^{U_{\min m a x}}$, respectively. To do this, we are going to use the following remark where, for some semiuninorms $U$, we express the value $\xi_{p, w}^{U}(A)$ (for subsets $A$ satisfying $\left.\mu_{|w|}(A)>|A| / n\right)$ as a function of $\mu_{p}(A) /|A|$ and $\mu_{|w|}(A) /|A|$.

Remark 2. Consider the four regions of the square $[0,1]^{2}$ (see Figure 2):

$$
R_{1}=[1 / n, 1]^{2}, \quad R_{2}=[0,1 / n) \times(1 / n, 1], \quad R_{3}=[0,1 / n]^{2}, \quad R_{4}=(1 / n, 1] \times[0,1 / n) .
$$

Given two weighting vectors $\boldsymbol{p}$ and $\boldsymbol{w}$, and $\varnothing \neq A \subsetneq N$, it is easy to check the following equivalences ${ }^{6}$ :

$$
\frac{\mu_{p}(A)}{|A|} \geq \frac{1}{n} \Leftrightarrow \frac{1-\mu_{p}(A)}{n-|A|} \leq \frac{1}{n}, \quad \frac{\mu_{|w|}(A)}{|A|} \geq \frac{1}{n} \Leftrightarrow \frac{1-\mu_{|w|}(A)}{n-|A|} \leq \frac{1}{n} .
$$

Therefore we have

$$
\left(\frac{\mu_{p}(A)}{|A|}, \frac{\mu_{|w|}(A)}{|A|}\right) \in R_{i} \Leftrightarrow\left(\frac{1-\mu_{p}(A)}{n-|A|}, \frac{1-\mu_{|w|}(A)}{n-|A|}\right) \in R_{i+2} \quad i \in\{1,2\} .
$$

\footnotetext{
${ }^{6}$ Note that if we only consider the equalities, the equivalences are also maintained.
} 
Now consider $\varnothing \neq A \subsetneq N$ with $\mu_{|w|}(A) /|A|>1 / n$. Then

$$
\xi_{p, w}^{U}(A)=1-(n-|A|) U\left(\frac{1-\mu_{p}(A)}{n-|A|}, \frac{1-\mu_{|w|}(A)}{n-|A|}\right) .
$$

In the following cases we can express the value $\xi_{p, w}^{U}(A)$ as a function of $\mu_{p}(A) /|A|$ and $\mu_{|w|}(A) /|A|$ (notice that in the last three cases it corresponds to a game associated with a SUOWA operator; that is, it coincides with $v_{p, w}^{U_{1}}(A)$ for some $\left.U_{1} \in \widetilde{\mathcal{U}}^{1 / n}\right)$.

1. If $\left(\mu_{p}(A) /|A|, \mu_{|w|}(A) /|A|\right) \in R_{1}$ and $\left.U\right|_{R_{3}}=0$, then $\xi_{p, w}^{U}(A)=1$.

2. If $\left(\mu_{p}(A) /|A|, \mu_{|w|}(A) /|A|\right) \in R_{1}$ and $\left.U\right|_{R_{3}}=\min$, or $\left(\mu_{p}(A) /|A|, \mu_{|w|}(A) /|A|\right) \in R_{2}$ and $\left.U\right|_{R_{4}}=\min$, then

$$
\xi_{p, w}^{U}(A)=1-(n-|A|) \min \left(\frac{1-\mu_{p}(A)}{n-|A|}, \frac{1-\mu_{|w|}(A)}{n-|A|}\right)=\max \left(\mu_{p}(A), \mu_{|w|}(A)\right) .
$$

3. If $\left(\mu_{p}(A) /|A|, \mu_{|w|}(A) /|A|\right) \in R_{2}$ and $\left.U\right|_{R_{4}}=\max$, then

$$
\xi_{p, w}^{U}(A)=1-(n-|A|) \max \left(\frac{1-\mu_{p}(A)}{n-|A|}, \frac{1-\mu_{|w|}(A)}{n-|A|}\right)=\min \left(\mu_{p}(A), \mu_{|w|}(A)\right) .
$$

4. If $\left(\mu_{p}(A) /|A|, \mu_{|w|}(A) /|A|\right) \in R_{2}$ and $\left.U\right|_{R_{4}}(x, y)=x+y-1 / n$, then

$$
\xi_{p, w}^{U}(A)=1-(n-|A|)\left(\frac{1-\mu_{p}(A)}{n-|A|}+\frac{1-\mu_{|w|}(A)}{n-|A|}-\frac{1}{n}\right)=\mu_{p}(A)+\mu_{|w|}(A)-\frac{|A|}{n}=|A|\left(\frac{\mu_{p}(A)}{|A|}+\frac{\mu_{|w|}(A)}{|A|}-\frac{1}{n}\right) .
$$

Proposition 2. Let $\boldsymbol{p}$ and $\boldsymbol{w}$ be two weighting vectors. Then $\xi_{p, \boldsymbol{w}}^{U_{\min }}=\xi_{p, \boldsymbol{w}}^{U_{\operatorname{mix}}^{\max }}=v_{p, \boldsymbol{w}}^{U_{\max }^{\max }}$.

Proof. Given $A \subsetneq N$ with $|A| \geq 1$, we distinguish two cases:

1. If $\mu_{|w|}(A) \leq|A| / n$, then it is obvious that $\xi_{p, w}^{U_{\min }^{\max }}(A)=v_{p, w}^{U_{\min }^{\max }}(A)$.

2. If $\mu_{|w|}(A)>|A| / n$, then $\left(\mu_{p}(A) /|A|, \mu_{|w|}(A) /|A|\right) \in R_{1} \cup R_{2}$, and $\left.U_{\min }^{\max }\right|_{R_{3} \cup R_{4}}=\min$. Therefore, by the second item of Remark 2, $\xi_{p, \boldsymbol{w}}^{U_{\min }^{\max }}(A)=\max \left(\mu_{p}(A), \mu_{|\boldsymbol{w}|}(A)\right)=v_{\boldsymbol{p}, \boldsymbol{w}}^{U_{\operatorname{mix}}^{\max }}(A)$.

Proposition 3. Let $\boldsymbol{p}$ and $\boldsymbol{w}$ be two weighting vectors. Then $\xi_{\boldsymbol{p}, \boldsymbol{w}}^{U_{\top}}=\xi_{\boldsymbol{p}, \boldsymbol{w}}^{U_{\max }}=\xi_{\boldsymbol{p}, \boldsymbol{w}}^{U_{\min m a x}}=v_{\boldsymbol{p}, \boldsymbol{w}}^{U_{\min }}$.

Proof. Given $A \subsetneq N$ with $|A| \geq 1$, we distinguish three cases:

1. If $\mu_{|w|}(A) \leq|A| / n$, then it is obvious that $\xi_{\boldsymbol{p}, \boldsymbol{w}}^{U_{\operatorname{minmax}}}(A)=v_{\boldsymbol{p}, \boldsymbol{w}}^{U_{\operatorname{minmax}}}(A)$.

2. If $\mu_{|w|}(A)>|A| / n$ and $\mu_{p}(A)<|A| / n$, then $\left(\mu_{p}(A) /|A|, \mu_{|w|}(A) /|A|\right) \in R_{2}$, and $\left.U_{\operatorname{minmax}}\right|_{R_{4}}=\max$. Therefore, by the third item of Remark $2, \xi_{\boldsymbol{p}, \boldsymbol{w}}^{U_{\operatorname{minmax}}}(A)=\min \left(\mu_{\boldsymbol{p}}(A), \mu_{|\boldsymbol{w}|}(A)\right)=v_{\boldsymbol{p}, \boldsymbol{w}}^{U_{\min m a x}}(A)$.

3. If $\mu_{|w|}(A)>|A| / n$ and $\mu_{p}(A) \geq|A| / n$, then $\left(\mu_{p}(A) /|A|, \mu_{|w|}(A) /|A|\right) \in R_{1}$, and $\left.U_{\operatorname{minmax}}\right|_{R_{3}}=\min$. Therefore, by the second item of Remark 2, $\xi_{\boldsymbol{p}, \boldsymbol{w}}^{U_{\operatorname{minmax}}}(A)=\max \left(\mu_{\boldsymbol{p}}(A), \mu_{|\boldsymbol{w}|}(A)\right)=v_{\boldsymbol{p}, \boldsymbol{w}}^{U_{\min m a x}}(A)$.

In the case of the semiuninorm $U_{T_{\mathbf{M}}}$ we also have the equality between the games $\xi_{\boldsymbol{p}, \boldsymbol{w}}^{U_{T_{\mathbf{M}}}}$ and $v_{\boldsymbol{p}, \boldsymbol{w}}^{U_{T_{\mathbf{M}}}}$.

Proposition 4. Let $\boldsymbol{p}$ and $\boldsymbol{w}$ be two weighting vectors. Then $\xi_{\boldsymbol{p}, \boldsymbol{w}}^{U_{T_{\mathbf{M}}}}=v_{\boldsymbol{p}, \boldsymbol{w}}^{U_{T_{\mathbf{M}}}}$. 
Proof. Given $A \subsetneq N$ with $|A| \geq 1$, we distinguish three cases:

1. If $\mu_{|\boldsymbol{w}|}(A) \leq|A| / n$, then it is obvious that $\xi_{\boldsymbol{p}, \boldsymbol{w}}^{U_{T_{\mathbf{M}}}}(A)=v_{\boldsymbol{p}, \boldsymbol{w}}^{U_{T_{\mathbf{M}}}}(A)$.

2. If $\mu_{|w|}(A)>|A| / n$ and $\mu_{p}(A)<|A| / n$, then $\left(\mu_{p}(A) /|A|, \mu_{|w|}(A) /|A|\right) \in R_{2}$, and $\left.U_{T_{\mathbf{M}}}\right|_{R_{4}}(x, y)=x+y-1 / n$. Therefore, by the fourth item of Remark 2 ,

$$
\xi_{p, w}^{U_{T_{\mathbf{M}}}}(A)=|A|\left(\frac{\mu_{p}(A)}{|A|}+\frac{\mu_{|w|}(A)}{|A|}-\frac{1}{n}\right)=v_{p, w}^{U_{T_{\mathbf{M}}}}(A) .
$$

3. If $\mu_{|w|}(A)>|A| / n$ and $\mu_{p}(A) \geq|A| / n$, then $\left(\mu_{p}(A) /|A|, \mu_{|w|}(A) /|A|\right) \in R_{1}$, and $\left.U_{T_{\mathrm{M}}}\right|_{R_{3}}=\min$. Therefore, by the second item of Remark 2, $\xi_{p, w}^{U_{T_{\mathbf{M}}}}(A)=\max \left(\mu_{\boldsymbol{p}}(A), \mu_{|\boldsymbol{w}|}(A)\right)=v_{\boldsymbol{p}, \boldsymbol{w}}^{U_{T_{\mathbf{M}}}}(A)$.

In addition to the previously established relationships (i.e., $\xi_{\boldsymbol{p}, \boldsymbol{w}}^{U_{\min }}=\xi_{\boldsymbol{p}, \boldsymbol{w}}^{U_{\operatorname{mix}}^{\max }}=v_{\boldsymbol{p}, \boldsymbol{w}}^{U_{\operatorname{mix}}^{\max }}, \xi_{\boldsymbol{p}, \boldsymbol{w}}^{U_{\top}}=\xi_{\boldsymbol{p}, \boldsymbol{w}}^{U_{\max }}=\xi_{\boldsymbol{p}, \boldsymbol{w}}^{U_{\operatorname{minmax}}}=v_{\boldsymbol{p}, \boldsymbol{w}}^{U_{\min m a x}}$, and $\xi_{\boldsymbol{p}, \boldsymbol{w}}^{U_{T_{\mathbf{M}}}}=v_{\boldsymbol{p}, \boldsymbol{w}}^{U_{T_{\mathbf{M}}}}$ for any weighting vectors $\boldsymbol{p}$ and $\boldsymbol{w}$ ) we can provide some new interesting relations when the weighting vectors $\boldsymbol{w}$ are nondecreasing or nonincreasing.

In the case of nondecreasing weighting vectors $\boldsymbol{w}$, we have $\sum_{i=1}^{j} w_{i} \leq j / n$ for all $j \in N$ (see Llamazares [34]). This means that $\mu_{|\boldsymbol{|}|}(A) \leq|A| / n$ for any nonempty subset $A$ of $N$, and consequently, $v_{p, w}^{U}=\xi_{p, w}^{U}$ for any weighting vector $\boldsymbol{p}$ and any semiuninorm $U$. Moreover, these games only uses the values of the semiuninorm in the region $[0,1] \times[0,1 / n]$. Therefore, semiuninorms defined in the same way in this region return the same games. Note also that, for any weighting vector $\boldsymbol{p}$, the games $v_{\boldsymbol{p}, \boldsymbol{w}}^{U}$ are capacities when $U=U_{\min }, U=U_{\min }^{\max }, U=U_{P}$ or $U=U_{\widetilde{P}}(\operatorname{see}$ Llamazares [34, 29, 4]).

In the case of nonincreasing weighting vectors $\boldsymbol{w}$, we have $\sum_{i=1}^{j} w_{i} \geq j / n$ for all $j \in N$ (see Llamazares [34]). This means that $\mu_{|w|}(A) \geq|A| / n$ for any nonempty subset $A$ of $N$, and consequently, the games $v_{p, w}^{U}$ only uses the values of the semiuninorms in the region $[0,1] \times[1 / n, n]$. So, semiuninorms defined in the same way in this region return the same games $v_{p, w}^{U}$; that is (see Table 1 ),

1. $v_{\boldsymbol{p}, \boldsymbol{w}}^{U_{\max }}=v_{\boldsymbol{p}, \boldsymbol{w}}^{U_{\operatorname{mix}}^{\max }}$, and both are capacities (see Llamazares [34]).

2. $v_{\boldsymbol{p}, \boldsymbol{w}}^{U_{\operatorname{minmax}}}=v_{\boldsymbol{p}, \boldsymbol{w}}^{U_{\min }}=v_{\boldsymbol{p}, \boldsymbol{w}}^{U_{\perp}}, v_{\boldsymbol{p}, \boldsymbol{w}}^{U_{T_{\mathbf{M}}}}=v_{\boldsymbol{p}, \boldsymbol{w}}^{U_{T_{\mathbf{L}}}}, v_{\boldsymbol{p}, \boldsymbol{w}}^{U_{P}}=v_{\boldsymbol{p}, \boldsymbol{w}}^{U_{\widetilde{w}}}$.

Regarding the games $\xi_{p, w}^{U}$, we have the following results.

Proposition 5. Let $\boldsymbol{w}$ be a nonincreasing weighting vector and let $U_{1}, U_{2} \in \widetilde{\mathcal{U}}^{1 / n}$ such that $U_{1} \leq U_{2}{ }^{7}$ Then $\xi_{\boldsymbol{p}, \boldsymbol{w}}^{U_{1}} \geq \xi_{\boldsymbol{p}, \boldsymbol{w}}^{U_{2}}$ for any weighting vector $\boldsymbol{p}$.

Proof. Given $A \neq \varnothing$, since $\mu_{|w|}(A) \geq|A| / n$, we have

$$
\xi_{\boldsymbol{p}, \boldsymbol{w}}^{U_{1}}(A)=1-(n-|A|) U_{1}\left(\frac{1-\mu_{\boldsymbol{p}}(A)}{n-|A|}, \frac{1-\mu_{|\boldsymbol{w}|}(A)}{n-|A|}\right) \geq 1-(n-|A|) U_{2}\left(\frac{1-\mu_{\boldsymbol{p}}(A)}{n-|A|}, \frac{1-\mu_{|\boldsymbol{w}|}(A)}{n-|A|}\right)=\xi_{\boldsymbol{p}, \boldsymbol{w}}^{U_{2}}(A) .
$$

Proposition 6. Let $\boldsymbol{w}$ be a nonincreasing weighting vector. Then $\xi_{p, w}^{U_{\perp}} \geq v_{p, w}^{U_{\top}}$ for any weighting vector $\boldsymbol{p}$.

\footnotetext{
${ }^{7}$ In fact, for the result to be true it is sufficient that the inequality be satisfied in the region $[0,1] \times[0,1 / n]$.
} 
Table 2: Values of some games in subsets of cardinality 1.

\begin{tabular}{cccccc}
\hline Set & $\xi_{\boldsymbol{p}, \boldsymbol{w}}^{U_{\tilde{\boldsymbol{w}}}}$ & $\xi_{\boldsymbol{p}, \boldsymbol{w}}^{U_{T_{\mathbf{L}}}}$ & $v_{\boldsymbol{p}, \boldsymbol{w}}^{U_{\top}}$ & $\xi_{\boldsymbol{p}, \boldsymbol{w}}^{U_{\min }}$ & $\xi_{\boldsymbol{p}, \boldsymbol{w}}^{U_{P}}$ \\
\hline$\{1\}$ & 0.6 & 0.65 & 0.5 & 0.5 & 0.5 \\
$\{2\}$ & 0.36 & 0.35 & 0.4 & 0.4 & 0.36 \\
$\{3\}$ & 0.36 & 0.35 & 0.4 & 0.4 & 0.36 \\
$\{4\}$ & 0.28 & 0.25 & 0.4 & 0.4 & 0.28 \\
\hline
\end{tabular}

Proof. Given $A \neq \varnothing$, since $\mu_{|w|}(A) \geq|A| / n$, we distinguish the following cases:

1. If $\mu_{|w|}(A)=|A| / n$, then $\xi_{p, w}^{U_{\perp}}(A)=v_{p, w}^{U_{\top}}(A)=\mu_{p}(A)$.

2. If $\mu_{|w|}(A)>|A| / n$ and $\mu_{p}(A)<|A| / n$, then $\left(\mu_{p}(A) /|A|, \mu_{|w|}(A) /|A|\right) \in R_{2}$, and $\left.U_{\perp}\right|_{R_{4}}=$ min. Therefore, by the second item of Remark 2, $\xi_{\boldsymbol{p}, \boldsymbol{w}}^{U_{\perp}}(A)=\max \left(\mu_{\boldsymbol{p}}(A), \mu_{|\boldsymbol{w}|}(A)\right)=v_{\boldsymbol{p}, \boldsymbol{w}}^{U_{\top}}(A)$.

3. If $\mu_{|w|}(A)>|A| / n$ and $\mu_{p}(A) \geq|A| / n$, then $\left(\mu_{p}(A) /|A|, \mu_{|w|}(A) /|A|\right) \in R_{1}$, and $\left.U_{\perp}\right|_{R_{3}}=0$. Therefore, by the first item of Remark $2, \xi_{p, w}^{U_{\perp}}(A)=1 \geq v_{p, w}^{U_{\top}}(A)$.

It is also worth noting that it is not possible to establish an order between $\xi_{\boldsymbol{p}, \boldsymbol{w}}^{U_{\widetilde{p}}}$ and $v_{\boldsymbol{p}, \boldsymbol{w}}^{U_{\top}}$ or $\xi_{\boldsymbol{p}, \boldsymbol{w}}^{U_{\min }}$; nor between $\xi_{\boldsymbol{p}, \boldsymbol{w}}^{U_{T_{\mathbf{L}}}}$ and the previous games or $\xi_{\boldsymbol{p}, \boldsymbol{w}}^{U_{P}}$. To see this, consider Table 2 where we show the values of the above games on the sets of cardinality 1 when $n=4, \boldsymbol{p}=(0.5,0.2,0.2,0.1)$, and $\boldsymbol{w}=(0.4,0.3,0.2,0.1)$.

The above comments on the games $v_{p, w}^{U}$ and $\xi_{p, w}^{U}$, along with those made in Remark 1, can be summarized in Figures $3 \mathrm{a}$ and $3 \mathrm{~b}$, where we show Hasse diagrams of the usual partial order between these games in the case of nondecreasing and nonincreasing weighting vectors $\boldsymbol{w}$ (being $U$ a semiuninorm of those considered in Table 1). Games in the same node means that they are the same game, and the blue color means that the games are capacities.

\section{The pointwise convergence of the games $v_{p, w}^{h}$ and $\xi_{p, w}^{h}$}

The study of the games $v_{p, \boldsymbol{w}}^{h}$ and $\xi_{\boldsymbol{p}, \boldsymbol{w}}^{h}$ has been carried out by Llamazares [20]. One of the results given there establishes condition under which the functions of the form $h(x)=-(f(x))^{-k}$ or $\tilde{h}(x)=-\mathrm{e}^{(f(x))^{-k}}$, where $k>0$, allow to obtain normalized capacities when $\boldsymbol{w}$ is unimodal. Moreover, seven types of functions, together with the conditions on $k$, were given to illustrate the variety of functions available.

In this section we are going to show that when $h$ is of one of the above forms, the games $v_{\boldsymbol{p}, \boldsymbol{w}}^{h}$ and $\xi_{\boldsymbol{p}, \boldsymbol{w}}^{h}$ converge pointwise to the game $v_{p, w}^{U_{\operatorname{mix}}^{\max }}$. To prove this, we first show the pointwise convergence of the semiuninorms $U_{h}^{\max }$ and $U_{\widetilde{h}}^{\max }$ to $U_{\min }^{\max }$ (notice that in the case of $U_{\widetilde{h}}^{\max }$ it is necessary to impose an additional condition on the function $f$ ).

Proposition 7. Let $f:[0,1] \longrightarrow[0, f(1)]$ be a strictly increasing bijection. Then, 


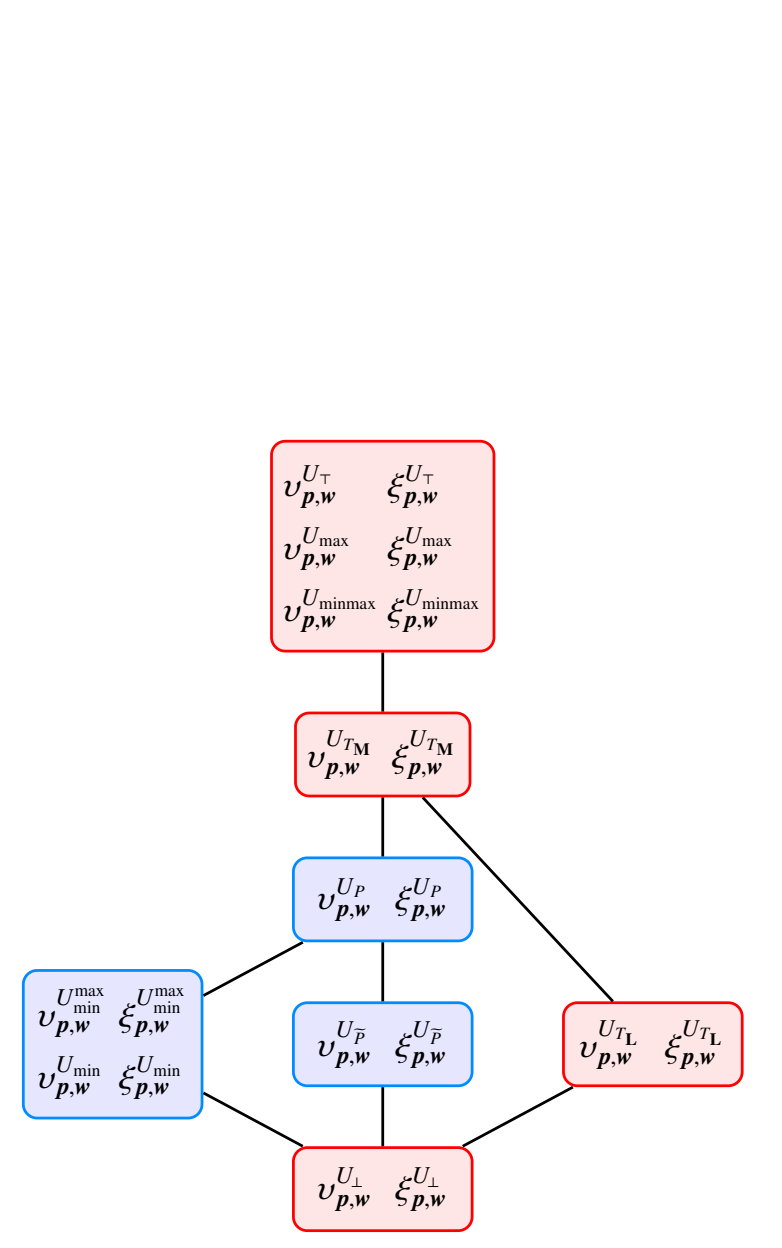

(a) Nondecreasing weighting vectors $\boldsymbol{w}$.

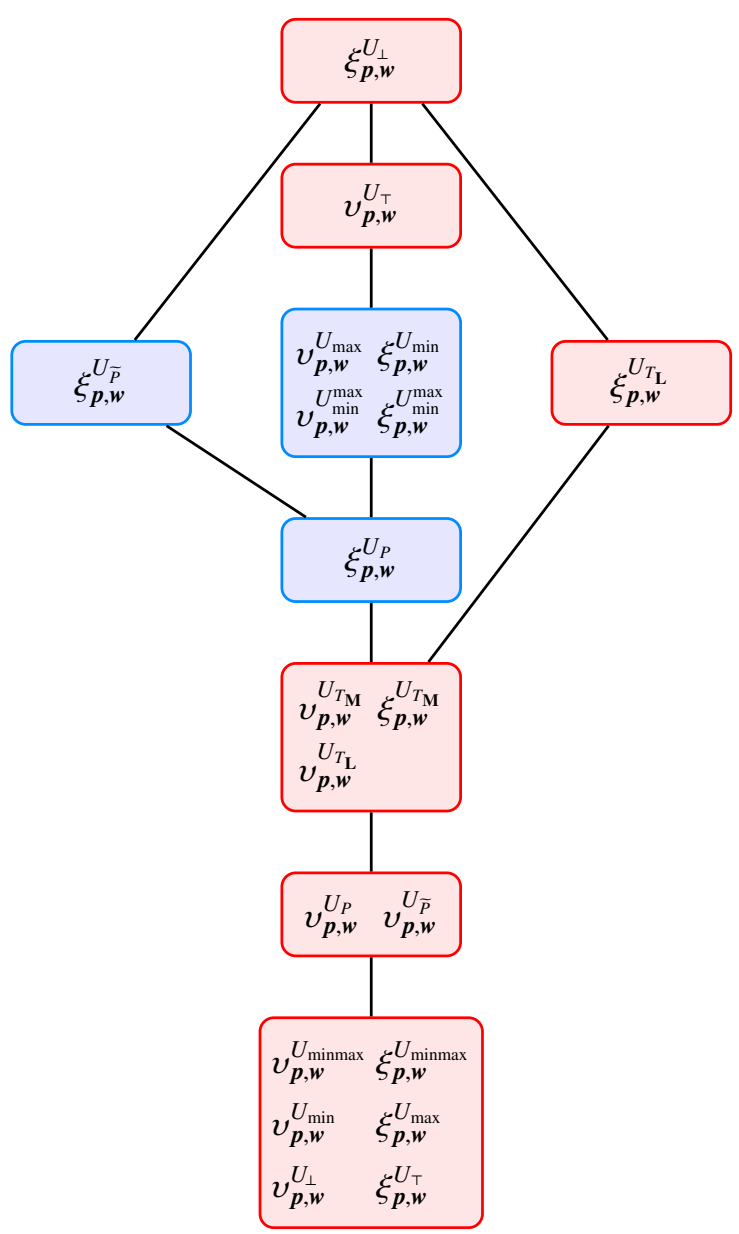

(b) Nonincreasing weighting vectors $\boldsymbol{w}$.

Figure 3: Hasse diagram showing the usual partial order between some games.

1. If $\left\{h_{m}\right\}_{m \in \mathbb{N}}$ is the sequence of functions defined by $h_{m}(x)=-(f(x))^{-m}$, then

$$
\lim _{m \rightarrow \infty} U_{h_{m}}^{\max }(x, y)=U_{\min }^{\max }(x, y),
$$

for any $(x, y) \in[0,1]^{2}$.

2. If $f(1 / n) \leq 1$ and $\left\{\widetilde{h}_{m}\right\}_{m \in \mathbb{N}}$ is the sequence of functions defined by $\widetilde{h}_{m}(x)=-\mathrm{e}^{(f(x))^{-m}}$, then

$$
\lim _{m \rightarrow \infty} U_{\widetilde{h}_{m}}^{\max }(x, y)=U_{\min }^{\max }(x, y)
$$

for any $(x, y) \in[0,1]^{2}$.

Proof. Let $f:[0,1] \longrightarrow[0, f(1)]$ be a strictly increasing bijection.

1. Consider the sequence of functions $\left\{h_{m}\right\}_{m \in \mathbb{N}}$ defined by $h_{m}(x)=-(f(x))^{-m}$ (notice that $h_{m}(0)=-\infty$ for any $m \in \mathbb{N})$. Given $(x, y) \in[0,1]^{2}$, we distinguish the following cases: 
(a) If $y \geq 1 / n$, then $U_{h_{m}}^{\max }(x, y)=U_{\min }^{\max }(x, y)$ for any $m \in \mathbb{N}$ and, consequently, the result is obvious.

(b) If $y<1 / n$ and $x y=0$ (i.e., $x=0$ or $y=0$ ), then $U_{h_{m}}^{\max }(x, y)=0=\min (x, y)=U_{\min }^{\max }(x, y)$ for any $m \in \mathbb{N}$ and, consequently, the result is obvious.

(c) If $y<1 / n$ and $x y \neq 0$, then

$$
U_{h_{m}}^{\max }(x, y)=f^{-1}\left(\left((f(x))^{-m}+(f(y))^{-m}-(f(1 / n))^{-m}\right)^{-1 / m}\right) .
$$

Since $f^{-1}$ is continuous and strictly increasing, to prove that

$$
\lim _{m \rightarrow \infty} U_{h_{m}}^{\max }(x, y)=U_{\min }^{\max }(x, y)=\min (x, y)
$$

is equivalent to show that

$$
\lim _{m \rightarrow \infty}\left(\frac{1}{(f(x))^{m}}+\frac{1}{(f(y))^{m}}-\frac{1}{(f(1 / n))^{m}}\right)^{1 / m}=\frac{1}{\min (f(x), f(y))} .
$$

But this result follows from the facts that

$$
\left(\frac{1}{(f(x))^{m}}+\frac{1}{(f(y))^{m}}-\frac{1}{(f(1 / n))^{m}}\right)^{1 / m}=\frac{1}{\min (f(x), f(y))}\left(1+\left(\frac{\min (f(x), f(y))}{\max (f(x), f(y))}\right)^{m}-\left(\frac{\min (f(x), f(y))}{f(1 / n)}\right)^{m}\right)^{1 / m}
$$

and that

$$
\lim _{m \rightarrow \infty}\left(1+\left(\frac{\min (f(x), f(y))}{\max (f(x), f(y))}\right)^{m}-\left(\frac{\min (f(x), f(y))}{f(1 / n)}\right)^{m}\right)^{1 / m}=1 .
$$

2. Consider the sequence of functions $\left\{\widetilde{h}_{m}\right\}_{m \in \mathbb{N}}$ defined by $\widetilde{h}_{m}(x)=-\mathrm{e}^{(f(x))^{-m}}$. Given $(x, y) \in[0,1]^{2}$, the cases $y \geq 1 / n$, and $y<1 / n$ with $x y=0$ can be proven by using the same arguments as in the previous item. Consider now the case $y<1 / n$ with $x y \neq 0$. Since

$$
U_{\widetilde{h}_{m}}^{\max }(x, y)=f^{-1}\left(\left(\log \left(\mathrm{e}^{(f(x))^{-m}}+\mathrm{e}^{(f(y))^{-m}}-\mathrm{e}^{(f(1 / n))^{-m}}\right)\right)^{-1 / m}\right),
$$

and $f^{-1}$ is continuous and strictly increasing, to prove that

$$
\lim _{m \rightarrow \infty} U_{\widetilde{h}_{m}}^{\max }(x, y)=U_{\min }^{\max }(x, y)=\min (x, y)
$$

is equivalent to show that

$$
\lim _{m \rightarrow \infty}\left(\log \left(\mathrm{e}^{(f(x))^{-m}}+\mathrm{e}^{(f(y))^{-m}}-\mathrm{e}^{(f(1 / n))^{-m}}\right)\right)^{1 / m}=\frac{1}{\min (f(x), f(y))} .
$$

Note that by using the notation

$$
\alpha=\frac{1}{\min (f(x), f(y))}, \quad \beta=\frac{1}{\max (f(x), f(y))}, \quad \text { and } \quad \gamma=\frac{1}{f(1 / n)},
$$

we have $\alpha \geq \beta$, and $\alpha>\gamma \geq 1$. Therefore,

$$
\begin{gathered}
\lim _{m \rightarrow \infty}\left(\log \left(\mathrm{e}^{(f(x))^{-m}}+\mathrm{e}^{(f(y))^{-m}}-\mathrm{e}^{(f(1 / n))^{-m}}\right)\right)^{1 / m}=\lim _{m \rightarrow \infty}\left(\log \left(\mathrm{e}^{\alpha^{m}}+\mathrm{e}^{\beta^{m}}-\mathrm{e}^{\gamma^{m}}\right)\right)^{1 / m} \\
=\lim _{m \rightarrow \infty}\left(\alpha^{m}+\log \left(1+\mathrm{e}^{-\alpha^{m}\left(1-(\beta / \alpha)^{m}\right)}-\mathrm{e}^{-\alpha^{m}\left(1-(\gamma / \alpha)^{m}\right)}\right)\right)^{1 / m}=\alpha .
\end{gathered}
$$


Taking into account the definition of the games $v_{\boldsymbol{p}, \boldsymbol{w}}^{h}$ and $\xi_{\boldsymbol{p}, \boldsymbol{w}}^{h}$, the following results are obvious.

Corollary 2. Let $\boldsymbol{p}$ and $\boldsymbol{w}$ be two weighting vectors and let $f:[0,1] \longrightarrow[0, f(1)]$ be a strictly increasing bijection. Then,

1. If $\left\{h_{m}\right\}_{m \in \mathbb{N}}$ is the sequence of functions defined by $h_{m}(x)=-(f(x))^{-m}$, then

$$
\lim _{m \rightarrow \infty} v_{\boldsymbol{p}, \boldsymbol{w}}^{h_{m}}(A)=v_{\boldsymbol{p}, \boldsymbol{w}}^{U_{\min }^{\max }}(A), \quad \text { and } \quad \lim _{m \rightarrow \infty} \xi_{\boldsymbol{p}, \boldsymbol{w}}^{h_{m}}(A)=\xi_{\boldsymbol{p}, \boldsymbol{w}}^{U_{\operatorname{mix}}^{\max }}(A)=v_{\boldsymbol{p}, \boldsymbol{w}}^{U_{\min }^{\max }}(A),
$$

for any $A \subseteq N$.

2. If $f(1 / n) \leq 1$ and $\left\{\widetilde{h}_{m}\right\}_{m \in \mathbb{N}}$ is the sequence of functions defined by $\widetilde{h}_{m}(x)=-\mathrm{e}^{(f(x))^{-m}}$, then

$$
\lim _{m \rightarrow \infty} v_{\boldsymbol{p}, \boldsymbol{w}}^{\widetilde{h}_{m}}(A)=v_{\boldsymbol{p}, \boldsymbol{w}}^{U_{\operatorname{mix}}^{\max }}(A), \quad \text { and } \quad \lim _{m \rightarrow \infty} \xi_{\boldsymbol{p}, \boldsymbol{w}}^{\widetilde{h}_{m}}(A)=\xi_{\boldsymbol{p}, \boldsymbol{w}}^{U_{\operatorname{mix}}^{\max }}(A)=v_{\boldsymbol{p}, \boldsymbol{w}}^{U_{\min }^{\max }}(A),
$$

for any $A \subseteq N$

\section{Concluding remarks}

Several procedures for constructing families of functions that simultaneously generalize weighted means and OWA operators have been suggested in recent years. In a recent paper, Llamazares [20] has introduced a new generalization, the Semi-SUOWA operators, which have a close relationship with SUOWA operators. For this reason, in this paper we have analyzed the relationships between some games associated with SUOWA and Semi-SUOWA operators and, in the case of nondecreasing and nonincreasing weighting vectors $w$, we have given simple Hasse diagrams showing the usual partial order between the games $v_{p, w}^{U}$ and $\xi_{p, w}^{U}$ (being $U$ a semiuninorm of those considered in Table 1). Moreover, we have established a condition on $U$ that guarantees the monotonicity of the game $\xi_{p, w}^{U}$ when $w$ is unimodal, and we have shown the pointwise convergence of the games $v_{p, w}^{h}$ and $\xi_{p, w}^{h}$ (for some specific cases of $h$ ) to the game $v_{p, w}^{U_{\min }^{\max }}$.

\section{Acknowledgements}

The author is grateful to two anonymous referees for their insightful comments that helped improve the paper. This work is partially supported by the Spanish Ministry of Economy and Competitiveness and ERDF (Project ECO201677900-P).

\section{References}

[1] R. R. Yager, On ordered weighted averaging operators in multicriteria decision making, IEEE Trans. Syst., Man, Cybern. 18 (1988) $183-190$.

[2] B. Roy, Double pondération pour calculer une moyenne: Pourquoi et comment?, RAIRO - Oper. Res. 41 (2007) 125-139.

[3] B. Llamazares, An analysis of some functions that generalizes weighted means and OWA operators, Int. J. Intell. Syst. 28 (2013) $380-393$.

[4] B. Llamazares, SUOWA operators: A review of the state of the art, Int. J. Intell. Syst. 34 (2019) 790-818. 
[5] G. Beliakov, Comparing apples and oranges: The weighted OWA function, Int. J. Intell. Syst. 33 (2018) 1089-1108.

[6] B. Llamazares, Constructing Choquet integral-based operators that generalize weighted means and OWA operators, Inform. Fusion 23 (2015) $131-138$.

[7] J.-L. Marichal, Tolerant or intolerant character of interacting criteria in aggregation by the Choquet integral, Eur. J. Oper. Res. 155 (2004) $771-791$.

[8] L. S. Shapley, A value for $n$-person games, in: H. Kuhn, A. W. Tucker (Eds.), Contributions to the Theory of Games, volume 2, Princeton University Press, Princeton, 1953, pp. 307-317.

[9] J.-L. Marichal, $k$-intolerant capacities and Choquet integrals, Eur. J. Oper. Res. 177 (2007) 1453-1468.

[10] B. Llamazares, On the orness of SUOWA operators, in: J. Gil-Aluja, A. Terceño Gómez, J. C. Ferrer-Comalat, J. M. Merigó-Lindahl, S. Linares-Mustarós (Eds.), Scientific Methods for the Treatment of Uncertainty in Social Sciences, volume 377 of Advances in Intelligent Systems and Computing, Springer, Cham, 2015, pp. 41-51.

[11] B. Llamazares, Closed-form expressions for some indices of SUOWA operators, Inform. Fusion 41 (2018) 80-90.

[12] B. Llamazares, SUOWA operators: An analysis of their conjunctive/disjunctive character, Fuzzy Sets Syst. 357 (2019) 117-134.

[13] B. Llamazares, An analysis of Winsorized weighted means, Group Decis. Negot. 28 (2019) 907-933.

[14] B. Llamazares, On the relationship between the Crescent Method and SUOWA operators, IEEE Trans. Fuzzy Syst. doi: 10.1109/TFUZZ.2019.2934937 (????).

[15] W. J. Dixon, Simplified estimation from censored normal samples, Ann. Math. Stat. 31 (1960) 385-391.

[16] H. Wainer, Robust statistics: A survey and some prescriptions, J. Educ. Stat. 1 (1976) 285-312.

[17] C. Labreuche, A formal justification of a simple aggregation function based on criteria and rank weights, in: DA2PL'2018, Poznan (Poland).

[18] B. Llamazares, A behavioral analysis of WOWA and SUOWA operators, Int. J. Intell. Syst. 31 (2016) $827-851$.

[19] L. Jin, R. Mesiar, R. R. Yager, Melting probability measure with OWA operator to generate fuzzy measure: the Crescent Method, IEEE Trans. Fuzzy Syst. 27 (2019) 1309-1316.

[20] B. Llamazares, Generalizations of weighted means and OWA operators by using unimodal weighting vectors, IEEE Trans. Fuzzy Syst. 28 (2020) 1961-1970.

[21] R. Mesiar, S. Borkotokey, L. Jin, M. Kalina, Aggregation functions and capacities, Fuzzy Sets Syst. 346 (2018) $138-146$.

[22] M. Maschler, B. Peleg, The structure of the kernel of a cooperative game, SIAM J. Appl. Math. 15 (1967) 569-604.

[23] M. Maschler, B. Peleg, L. S. Shapley, The kernel and bargaining set for convex games, Int. J. Game Theory 1 (1971) $73-93$.

[24] G. Choquet, Theory of capacities, Ann. Inst. Fourier 5 (1953) 131-295.

[25] D. Denneberg, Non-Additive Measures and Integral, Kluwer Academic Publisher, Dordrecht, 1994.

[26] M. Grabisch, J. Marichal, R. Mesiar, E. Pap, Aggregation Functions, Cambridge University Press, Cambridge, 2009.

[27] B. Llamazares, Choosing OWA operator weights in the field of Social Choice, Inform. Sci. 177 (2007) $4745-4756$.

[28] X. Liu, A review of the OWA determination methods: Classification and some extensions, in: R. R. Yager, J. Kacprzyk, G. Beliakov (Eds.), Recent Developments in the Ordered Weighted Averaging Operators: Theory and Practice, Springer, Berlin, 2011, pp. 49-90.

[29] B. Llamazares, Construction of Choquet integrals through unimodal weighting vectors, Int. J. Intell. Syst. 33 (2018) 771-790.

[30] R. R. Yager, Centered OWA operators, Soft Comput. 11 (2007) 631-639.

[31] H.-W. Liu, Semi-uninorms and implications on a complete lattice, Fuzzy Sets Syst. 191 (2012) $72-82$.

[32] R. R. Yager, A. Rybalov, Uninorm aggregation operators, Fuzzy Sets Syst. 80 (1996) 111-120.

[33] B. Llamazares, A study of SUOWA operators in two dimensions, Math. Probl. Eng. 2015 (2015) Article ID 271491, 12 pages.

[34] B. Llamazares, SUOWA operators: Constructing semi-uninorms and analyzing specific cases, Fuzzy Sets Syst. 287 (2016) 119-136.

[35] E. P. Klement, R. Mesiar, E. Pap, Triangular Norms, volume 8 of Trends in Logic, Studia Logica Library, Kluwer Academic Publishers, Dordrecht, 2000 